\title{
Physical Exercise as the Spice of Life: The Impact of Regular Exercise on Anxiety and the Meaning of Life in Diabetes, Psoriasis, and Heart Disease Patients
}

\author{
Eirini Karakasidou*, Georgia Raftopoulou, Konstantinos Daoultzis, Konstantinos Kanellakis \\ Panteion University, Athens, Greece \\ Email: *irenekarakasidou@yahoo.com
}

How to cite this paper: Karakasidou, E., Raftopoulou, G., Daoultzis, K., \& Kanellakis, K. (2020). Physical Exercise as the Spice of Life: The Impact of Regular Exercise on Anxiety and the Meaning of Life in Diabetes, Psoriasis, and Heart Disease Patients. Psychology, 11, 2081-2101. https://doi.org/10.4236/psych.2020.1112130

Received: November 14, 2020

Accepted: December 28, 2020

Published: December 31, 2020

Copyright $\odot 2020$ by author(s) and Scientific Research Publishing Inc. This work is licensed under the Creative Commons Attribution International License (CC BY 4.0).

http://creativecommons.org/licenses/by/4.0/

\begin{abstract}
The primary scope of the current study was to investigate the effects of physical activity among three types of diseases, that is diabetes, psoriasis and heart disease in well-being and negative emotional states. The sample consisted of 120 Greek adults with a clinical diagnosis of a medical condition, aged between 30 and 50 years. The participants completed four self-report questionnaires (DASS 21, Meaning in Life, Subjective Happiness Scale, Life Satisfaction Scale) and reported their frequency of exercise and their demographic characteristics. A 3 (no, moderate, high exercise) $\times 3$ (diabetes, psoriasis, heart disease) factorial ANOVA was conducted. The results showed that regarding anxiety, symptoms were prospectively associated with lower exercise frequency. The findings suggest the prudence of exercising on psychological prosperity in patients with chronic disease.
\end{abstract}

\section{Keywords}

Well-Being, Negative Emotional State, Exercise, Medical Condition

\section{Introduction}

Over the previous years, exercise is accounted for to be a developing wellbeing issue everywhere throughout the world (Evans, Rohan, Howard, Ho, Dubbert, \& Stetson, 2017; Epps, To, Liu, Karanjit, \& Warren, 2019). Exercise may act protectively in reducing negative emotional state (NES) (Stubbs et al., 2017; Gordon, McDowell, Lyons, \& Herring, 2017). It has additionally been demonstrated that dysfunctional anxiety might be the cause of disruption, and it might also 
exhaust the person, both psychologically and physically (Bernstein and Borchardt, 1991). Regarding stress, it may influence performance negatively (Cohen, 2011). Furthermore, depressive symptoms result in significant consequences in one's life, deteriorating functionality, as well as negative emotions and dysfunctional self-regulation (Cotman \& Berchtold, 2002). Worldwide, people have to deal with the outcomes of NES daily and experience several consequences on their medical and psychological condition and ultimately in their well-being. Numerous research suggests that exercise has a buffering effect on anxiety, but at the same time promotes well-being in diabetes, heart disease and psoriasis patients. Well-being is a positive state of a person of being comfortable, happy and healthy, nurturing their skills and expressing their inner potential to the most possible extent. Several scientists have conducted research that has shown the beneficial effects of exercise on well-being (Mandolesi, Polverino, Montuori, Foti, Ferraioli, Sorrentino, \& Sorrentino, 2018; Rogerson, Wood, Pretty, Schoenmakers, Bloomfield, \& Barton, 2020). Furthermore, studies have focused on examining changes in anxiety and well-being according to the frequency of exercise in a susceptible population, demonstrating inconsistent results.

The first factor under investigation is the frequency of exercise. During the past years, the beneficial effects of exercise on the NES have been found in several studies. De Moor, Beem, Stubbe, Boomsma, and De Geus (2006) conducted comprehensive population-based research $(N=19,288)$, with adult and adolescent twins and their families to investigate whether regular physical activity has an effect on anxiety and depression. They presented evidence that regular exercise is associated with lower anxiety and depression in the population. Hassmén, Koivula, \& Uutela (2000) conducted a population study in Finland among 3403 individuals to explore the relationship between exercise frequency and psychological wellness. The findings revealed that participants who exercised at a moderate frequency benefited most of the positive effects of physical activity with regards to their well-being. Marquez, Jerome, Mc Auley, Snook and Canaklisova (2010) studied the impact of manipulated self-efficacy exercise in anxiety in a sample of sedentary women. Results reported that anxiety increased in the low efficacy condition, in the low-frequency exercisers.

Physical activity, apart from the psychological well-being, can also have a positive effect on mental health (Penedo \& Dahn, 2005). Scully, Kremer, Meade, Graham and Dudgeon (1998) also conducted a critical review regarding the relationship between exercise and psychological well-being. The findings revealed that those who exercise at a moderate pace and frequency could benefit both physically and psychologically. However, attention is drawn to the type as well as the frequency of exercise. Different types of exercise should be suggested for each individual with regards to their personal physical and emotional state. What is more, excessive exercise could attribute to the opposite results.

Studies have investigated the impact of exercise on NES, apart from general population also to susceptible one (De Moor et al., 2008; Ströhle, 2009; Weinstein, 
Maayan, \& Weinstein, 2015). With respect to psychological disorders, Herring, O'Connor, and Dishman (2010) conducted a systematic review on the association of physical activity among clinically diagnosed sample with anxiety symptoms. It revealed that exercise reduced the latter. Additionally, a systematic review of physical activity regarding anxiety disorders (Jayakody, Gunadasa and Hosker, 2014) revealed that exercise is beneficial. However, it does not appear to be as effective as an antidepressant therapy. Research regarding the association of compulsive exercise, depression and anxiety (Weinstein, Maayan and Weinstein, 2015) among professional and recreational participants, showed evidence that those who exercise, use the physical activity in order to deal with anxiety and depression. They also revealed that excessive or compulsory exercise might have the opposite effects. With respect to medical conditions, the frequency and quality of physical exercise have been studied numerous times in patient samples (De Moor et al., 2008; Ströhle, 2009; Weinstein, Maayan, and Weinstein, 2015). The selection of medical conditions is random, comparing potentially different effects of exercise and NES on different types of diseases (liver, skin and heart diseases).

The second factor under investigation is type of disease. Diabetes, psoriasis and heart disease are of importance, due to their high prevalence rates in the Greek population. The prevalence of diabetes in Greece is $7.4 \%$ among the adult population (International Diabetes Foundation, 2020). The prevalence of psoriasis is 1\% - 3\% (National Psoriasis Foundation, 2020). Heart disease accounts for almost $45 \%$ of all deaths and leads to significant morbidity (Michas, Karvelas, \& Trikas, 2019). These three types of diseases carry an increased risk of psychological distress. At this early stage, it seems that by studying and understanding this concept, it is possible to create such interventions that allow the prevention or reduction of problems in people's lives.

Many studies and systematic reviews have been conducted investigating physical exercise and negative emotional state in an array of diseases. It was found that there is an interaction between physical exercise and negative emotional states in an array of diseases, such as mental disorders (Cohen \& Rodriguez, 1995), heart disease (Rogerson, Murphy, Bird, \& Morris, 2012) anxiety disorders (Cotman \& Berchtold, 2002; Ströhle, 2009) and depression (Cotman \& Berchtold, 2002). Cohen and Rodriguez (1995) conducted a systematic review in order to support that there is an interaction between physical activity and NES. Ströhle (2009) reviewed the literature in order to present evidence that physical activity has a relationship with lower levels of depression and that exercise might be useful as a therapeutic tool for depression and anxiety disorders. Additionally, Cotman and Berchtold (2002) supported that those who have anxiety and depression disorders may benefit from moderate physical activity. In this study, we focused on diabetes, psoriasis and heart disease.

Diabetes is a chronic disease that occurs either when the pancreas does not produce enough insulin or when the body cannot effectively use the insulin it 
produces. Insulin is a hormone that regulates blood sugar. Hyperglycaemia, or raised blood sugar, is a common effect of uncontrolled diabetes and over time leads to severe damage to many of the body's systems, especially the nerves and blood vessels. It affects more than 400 million people worldwide (Genuth, Alberti, Bennett, Buse, Defronzo, Kahn, Kitzmiller, Knowler, Lebovitz, Lernmark, Nathan, Palmer, Rizza, Saudek, Shaw, Steffes, Stern, Tuomilehto and Zimmet, 2003). Diabetes patients need to maintain healthy body weight, thus being physically active in order to face the effects of the disease. It is also supported that exercise may increase well-being among diabetes patients (Reid et al., 2010). However, they do not adhere to a systematic exercise routine. On the other hand, they deal with more psychological distress than the average population (Ivanova, Burns, Deschênes, Knäuper, \& Schmitz, 2017). It is conducted a systematic review to investigate the psychological effects of exercise among type 2 diabetes. Their findings supported that overall well-being was increased. It is also suggested that regarding diabetes, there is a decreasing effect of exercise on the negative emotional state (Huebschmann, Schauer, Bauer, Regensteiner, \& Reusch, 2018).

Psoriasis is a chronic inflammatory hyperproliferative disease of the skin, scalp, nails, and joints. The physical symptoms of psoriasis cause psychological distress, especially as a result of stigmatisation, self-consciousness, and embarrassment, which can, in turn, affect social activities, such as exercising (Cunliffe et al., 2003). Regarding psoriasis, although there is evidence that psoriasis patients suffer from psychological distress (De Arruda \& De Moraes, 2001; Van Voorhees \& Fried, 2009), there is no research regarding the effect of physical activity on well-being among this population. However, there is evidence that supports that psoriasis patients avoid exercising possibly for both psychological and physiological reasons (Torres, Alexandre, Mendonça, Vasconcelos, Silva, \& Selores, 2014). Relatively high rates of depression are reported in patients with psoriasis. Managing psoriasis results in an improved health-related quality of life, thus to an improvement of physical and psychological aspects. In a study conducted by McDonough and his colleagues (2014), it was demonstrated that psoriasis patients show significantly higher depressive and anxiety symptoms, which are associated with disease-related factors. Therefore, it seems necessary to investigate whether exercise may support patients to enhance their psychological well-being.

Heart disease includes a range of diseases that affect the heart. Diseases that can cause heart disease include blood vessel diseases such as coronary heart disease, heart rhythm problems (arrhythmias), and congenital heart damage. Cardiovascular disease is generally associated with conditions that include narrowed or blocked blood vessels that can lead to heart attack, chest pain or stroke. Heart disease such as those that affect the heart muscle, valves or heart rate is also considered forms of heart disease. Many forms of heart disease can be prevented or treated with healthy lifestyle changes, such as exercise. Heart disease patients may also benefit from physical activity regarding well-being. In a meta-analysis 
study conducted by Liu and his partners (2018), there was evidence that Tai Chi may improve significantly psychosocial well-being among coronary heart disease patients. However, further investigation is needed because of the small number of studies that were reviewed. In addition, van der Mheen and his partner (2018) presented evidence that exercise may increase well-being. Whooley and his partners (2008) conducted a cohort study and showed that in a sample of outpatients with coronary heart disease, the association between depressive symptoms and adverse cardiovascular events was explained mainly by behavioural factors, particularly physical inactivity.

\section{The Current Study}

The current study aims to investigate the effects of physical activity among three types of diseases, that is diabetes, psoriasis and heart disease in well-being and negative emotional states. There has not been significant evidence for clinically diagnosed patients, that suffer from either heart condition, psoriasis or diabetes mellitus. Taking into account all of the above studies, some queries arise regarding the way these symptoms may be faced so that well-being may be improved. Related studies have investigated negative emotional state, both on healthy and susceptible population (De Moor et al., 2008; Ströhle, 2009; Weinstein, Maayan, and Weinstein, 2015). Thus, the primary purpose of this study is to explore whether there is a relation between the frequency of exercise and negative emotional state in a sample of patients with chronic disease. Secondary results include the relationship between physical activity, disease and well-being, as well as the interaction between type of disease, exercise and negative emotional state or well-being. This study involves patients with a medical record on the heart condition, diabetes mellitus and psoriasis. The significance of such an investigation is evident given that exercise may affect diabetes, psoriasis and heart disease, which seem to interact and influence negative emotional state and psychological well-being. Therefore, it is crucial to investigate further if physical activity has an effect on those symptoms. If exercise could in any way contribute to the improvement of quality of life in these patients, these results could provide new insights and foster existing intervention programs and tools for clinical populations, minimising NES. This investigation is essential so that we can increase our understanding of the role that exercise play in reducing anxiety symptoms and improve well-being in diabetes, psoriasis or heart disease. This may also be beneficial in developing well-informed intervention plans, to target specific susceptible population with chronic disease.

The study's hypotheses are as follows:

$\mathrm{H}_{1}$ : It is hypothesised that there will be at least one significant difference in negative emotional state and well-being in terms of the type of disease and/or frequency of exercise.

$\mathrm{H}_{3}$ : It is hypothesised that there will be an interaction between physical activity and medical condition in terms of negative emotional state or well-being. 


\section{Method}

\subsection{Design}

A two-way, factorial Analysis of Variance-ANOVA was implemented to investigate whether physical activity and the medical condition has an effect on symptoms of negative emotional state and well-being values. The dependent variables are symptoms of negative emotional state and well-being values: life satisfaction, presence of meaning in life, search in meaning in life and subjective happiness. Two-way ANOVA provides information regarding the mean and the standard deviation. Therefore, we ran tests of between-subjects effects as two independent variables is medical condition and frequency of exercise. We have run tests of between-subjects effects in order to explore the differences in the dependent variables between 3 different medical condition groups, who exercise at a different frequency (no physical activity, moderate frequency of physical activity, higher frequency of physical activity).

\subsection{Participants}

In this study, a sample of one-hundred-twenty $(\mathrm{N}=120)$ Greek adults participated, coming from a large and diverse pool of the general population. The sample consisted of 28 males, and 92 females, with an age range from 30 to 50 years old $(M=39.44, S D=8.16)$. The participants were separated into three groups, according to their medical condition, heart disease $(n=40)$, diabetes mellitus ( $\mathrm{n}$ $=40)$, or psoriasis $(\mathrm{n}=40)$. The sample was also divided into three groups: no exercisers ( $n=48)$, moderate frequency exercisers ( 1 or 2 times per week), ( $=$ $47)$ and high-frequency exercisers ( 3,4 or more times per week), ( $\mathrm{n}=25)$. Demographic characteristics of participants are reported below in Table 1. The current study compromises ethically to the ethics of Psychology. Participants provided volitional consent and method of recruitment was snowball sampling. Private data, except those required for the study, are anonymous as no identifying information was collected. Participants were required to fill in the DASS 21, Meaning in Life, Subjective Happiness Scale, Life Satisfaction Scale distributed in the format of Google form through emails and Facebook.

\subsection{Materials}

For the generation of the data, self-report questionnaires were administered, that recorded the following variables: demographic characteristics, Subjective Happiness Scale, Meaning in Life Questionnaire, DASS 21 and Life Satisfaction Questionnaire. The questionnaires were used to explore whether these factors have an effect primarily on their negative emotional state symptoms and secondly on their well-being.

Medical status was measured in each study. Individuals answered whether they clinically express one of the diseases: diabetes mellitus, heart disease or psoriasis ("Yes" or "No"). If the participants agreed, further information was gathered. The 
Table 1. Demographic characteristics of the participants $(\mathrm{N}=120)$.

\begin{tabular}{|c|c|c|c|c|c|}
\hline Demographic variables & $\begin{array}{l}\text { Psoriasis patients } \\
\qquad(N=40)\end{array}$ & $\begin{array}{l}\text { Diabetes patients } \\
\qquad(N=40)\end{array}$ & $\begin{array}{c}\text { Heart disease } \\
\text { patients }(N=40)\end{array}$ & $F, \chi^{2}$ & $P$ value \\
\hline Age, Mean (SD) & $39.55(8.01)$ & $38.75(8.35)$ & $40.03(8.28)$ & 0.25 & 0.782 \\
\hline Gender & & & & 1.77 & 0.452 \\
\hline Male & $9(22.5 \%)$ & $7(17.5 \%)$ & $12(30 \%)$ & & \\
\hline Female & $31(77.5 \%)$ & $33(82.5 \%)$ & $28(70 \%)$ & & \\
\hline Marital status & & & & 15.11 & 0.020 \\
\hline Single & $12(30 \%)$ & $18(45 \%)$ & $13(32 \%)$ & & \\
\hline Relationship & $3(75 \%)$ & - & $2(5 \%)$ & & \\
\hline Married & $25(62.5 \%)$ & $19(47.5 \%)$ & $19(47.5 \%)$ & & \\
\hline Divorced & - & $1(2.5 \%)$ & $6(15 \%)$ & & \\
\hline Widow & - & $2(100 \%)$ & - & & \\
\hline Education & & & & 6.93 & 0.269 \\
\hline Junior high school & $1(2.5 \%)$ & $2(5 \%)$ & $5(12.5 \%)$ & & \\
\hline High School & $13(32.5 \%)$ & $11(27.5 \%)$ & $15(37.5 \%)$ & & \\
\hline College student & $26(65 \%)$ & $25(62.5 \%)$ & $20(50 \%)$ & & \\
\hline University Graduate & - & $2(5 \%)$ & - & & \\
\hline Employment & & & & 10.67 & 0.0043 \\
\hline Employed & $30(75 \%)$ & $22(55 \%)$ & $31(77.5 \%)$ & & \\
\hline Unemployed & $10(25 \%)$ & $15(37.5 \%)$ & $5(12.5 \%)$ & & \\
\hline Retired & - & $2(50 \%)$ & - & & \\
\hline Housekeeping & - & $1(2.5 \%)$ & $2(5 \%)$ & & \\
\hline Medication & & & & 25.83 & 0.000 \\
\hline On medication & $14(35 \%)$ & $36(90 \%)$ & $22(55 \%)$ & & \\
\hline No medication & $26(65 \%)$ & $4(10 \%)$ & $18(45 \%)$ & & \\
\hline
\end{tabular}

demographic questionnaire includes details like age, gender, personal status, education level, employment status, details about their exercise routine and medication. Variables reflecting anxiety, stress, depression, subjective happiness, meaning in life and life satisfaction were measured.

$D A S S$-21. Depression, anxiety and stress symptoms were assessed with the short version of the DASS 21 (DASS 21, 21 items, $\alpha=0.965$ ). Their Cronbach alphas were $\mathrm{a}=0.84, \mathrm{a}=0.84$ and $\mathrm{a}=0.85$, respectively. In this study, Cronbach alphas were measured $\mathrm{a}=0.94, \mathrm{a}=0.89$ and $\mathrm{a}=0.91$. DASS 21 was originated by Lovibond and Lovibond (1995). It was used to evaluate the symptoms of anxiety, stress and depression, each self-reported. The standardisation was conducted by Pezirkianidis, Karakasidou, Lakioti, Stalikas and Galanakis (2018). The scale uses 21 statements on a four-point Likert scale (0: Did not apply to me at all, 1, 2, 3: "Applied to me very much or most of the time"), which shows to which level 
each statement applies to them during the previous week. The DASS 21 measures three scales: depression, anxiety and stress and has good psychometric properties. The standardisation of the Greek version of DASS showed satisfactory reliability and validity and the factorial structure of the scale was found to match the ones from previous studies of other countries.

SHS. The Subjective Happiness Scale documents a subjective perception of happiness, as the individual experiences, (SHS, four items, $\alpha=0.94$ ), (Lyubomirsky \& Lepper, 1999). The Cronbach alpha was $\alpha=0.76$. In this study, the Cronbach alpha was measured $\mathrm{a}=0.77$. It has been standardised in Greece by Karakasidou, Pezirkianidis, Stalikas and Galanakis (2016) and it showed satisfactory reliability and validity and the factorial structure of the scale was found to match the ones from previous studies of other countries. It was used to examine the subjectivity of an individual's global happiness. Its four items are rated on a 7-point Likert scale with lowest scores indicating not a very happy person and highest scores indicating a very happy person.

Meaning in life. Meaning in Life Questionnaire measures the levels of meaning in an individual's life, as well as the levels of searching meaning in life (MLQ, ten items, $\alpha=0.83$ for, $\alpha=0.8$ for; Steger, Frazier, Oishi, \& Kaler, 2006). The Cronbach alpha for the scale was $a=0.76$, for the Presence subscale, $a=0.83$ and the Search subscale was $\mathrm{a}=0.82$. The Cronbach alphas were calculated: $\mathrm{a}=0.84$, $\mathrm{a}=0.70$ and $\mathrm{a}=0.90$, respectively. It has been standardised with satisfactory reliability in the Greek sample population by Pezirkianidis and his partners (2016). The factorial structure of the scale was found to match the ones from previous studies of other countries. It consists of 10 items, rated on a 7-point Likert scale. It ranges from 1: "Absolutely True" to 7: to "Absolutely Untrue". The Presence of Meaning (e.g., "I understand my life's meaning.") and the Search for Meaning subscales (e.g., "I am seeking a purpose or mission for my life") consist of five items each.

Life satisfaction. Life Satisfaction Questionnaire measures the level of satisfaction that an individual draw from their life, using a 7-point Likert scale ranging from "Strongly Disagree" to "Strongly Agree (e.g., "So far I have gotten the important things I want in life."). (SWLS, five items, $\alpha=0.84$ ). Its Cronbach alpha was $\mathrm{a}=0.84$. The Cronbach alpha of this study was $\mathrm{a}=0.94$. It has been standardised in Greek sample population by Galanakis, Lakioti, Pezirkianidis, Karakasidou and Stalikas (2017) and it showed satisfactory reliability and validity and the factorial structure of the scale was found to match the ones from previous studies of other countries. The results show a continuum.

\subsection{Procedure}

The participants were recruited on the internet, using electronic tools. All individuals are voluntary participants. After agreeing to participate in this study, individuals were required to complete a demographic questionnaire, DASS 21, Subjective Happiness Scale, Meaning in Life Questionnaire and Life Satisfaction 
Questionnaire. Individuals were written notified about their rights. They were also notified regarding the type as well as the design of this study. If an individual wished to withdraw, there were no consequences. The data generated remain protected, and anonymity is guaranteed. Further information regarding the study was encouraged at any time. Assistance contact was also provided in case any discomfort would be caused. Each individual was provided with an e-version of the questionnaires as described above in the section of materials. Completion of the whole procedure lasted less than thirty minutes. During the debriefing, the aim of the study was presented.

\section{Results}

The responses of the questionnaires were recorded for each participant. Means $(M)$ and Standard deviations (S.D.) were calculated depending on the frequency of exercise and type of disease and are displayed in Table 2 below. For this study, the results from raw data that were collected through the questionnaires were analysed and calculated for all participants, and they are presented in Tables 2-5. A two-way, factorial Analysis of Variance-ANOVA was implemented to investigate whether physical activity and the medical condition has an effect on symptoms of negative emotional state and well-being values. Two way ANOVA provides information regarding the mean and the standard deviation. Therefore, we ran tests of between-subjects effects as there were two independent variables that are medical condition and frequency of exercise. Statistical

Table 2. Means, standard deviations as a function of 3 (Exercise) $\times 3$ (Type of disease) in DASS.

\begin{tabular}{|c|c|c|c|c|c|c|c|c|c|c|}
\hline \multirow{2}{*}{$\begin{array}{l}\text { Anxiety } \\
\text { Exercise }\end{array}$} & \multicolumn{2}{|c|}{ Psoriasis } & \multicolumn{2}{|c|}{ Diabetes } & \multicolumn{2}{|c|}{ Heart Disease } & \multicolumn{4}{|c|}{ ANOVA } \\
\hline & $\mathbf{M}$ & SD & $\mathbf{M}$ & SD & $\mathbf{M}$ & SD & Effect & F & df & $\eta^{2}$ \\
\hline No & 10.94 & 10.27 & 18.12 & 9.56 & 12.13 & 9.02 & $\mathrm{D}$ & $3.25^{\star}$ & 2 & 0.055 \\
\hline Moderate & 13.26 & 13.26 & 23.47 & 15.07 & 20.92 & 9.65 & E & $5.59^{* *}$ & 2 & 0.092 \\
\hline High & 7.5 & 6.61 & 9.78 & 7.71 & 15.33 & 10.70 & $\mathrm{D} \times \mathrm{E}$ & 1.25 & 4 & 0.043 \\
\hline \multicolumn{11}{|l|}{ Depression } \\
\hline Exercise & $\mathbf{M}$ & SD & $\mathbf{M}$ & SD & $\mathbf{M}$ & SD & Effect & F & df & $\eta^{2}$ \\
\hline No & 14.12 . & 12.24 & 12.75 & 12.88 & 12.00 & 10.28 & $\mathrm{D}$ & 0.635 & 2 & 0.011 \\
\hline Moderate & 15.79 & 12.54 & 22.40 & 2.63 & 19.69 & 10.86 & E & 2.709 & 2 & 0.047 \\
\hline High & 11.00 & 7.39 & 10.67 & 7.94 & 17.50 & 13.57 & $\mathrm{D} \times \mathrm{E}$ & 1.107 & 4 & 0.038 \\
\hline \multicolumn{11}{|l|}{ Stress } \\
\hline Exercise & $\mathbf{M}$ & $\mathrm{SD}$ & $\mathbf{M}$ & SD & $\mathbf{M}$ & SD & Effect & F & df & $\eta^{2}$ \\
\hline No & 17.18 & 11.11 & 23.25 & 11.57 & 16.00 & 9.13 & $\mathrm{D}$ & 1.650 & 2 & 0.029 \\
\hline Moderate & 20.21 & 10.87 & 26.13 & 12.41 & 22.31 & 11.51 & E & 2.596 & 2 & 0.045 \\
\hline High & 13.00 & 6.00 & 14.89 & 7.42 & 24.50 & 8.05 & $\mathrm{D} \times \mathrm{E}$ & 2.241 & 4 & 0.075 \\
\hline
\end{tabular}

${ }^{*}$ Significant at the $p<0.05$ level. ${ }^{*}$ Significant at the $p<0.01$ level. Diabetes mellitus patients, who exercise at a moderate frequency scored highest in anxiety (mean $=23.47, \mathrm{SD}=15.07) . \mathrm{E}=$ exercise, $\mathrm{D}=$ disease. 
Package for Social Science (SPSS) was used for the analysis of the results.

\subsection{DASS (Anxiety, Depression and Stress)}

Three $3 \times 3$ ANOVAs were conducted, after testing for their statistical assumptions. For anxiety, there seems to be a main effect of exercise $(p<0.05)$ and the main effect of a medical condition $(p<0.05)$ in anxiety. The tests of between-subjects effects showed at least one significant difference in anxiety between the 3 exercise groups: $F(2,111)=5.593, p<0.05, \eta^{2}=0.092$. The Anova also showed that there was a main effect of disease on anxiety: $F(2,111)=3.248, p$ $<0.05, \mathrm{Eta}^{2}=0.055$. No interaction between the type of disease and exercise was found. Diabetes mellitus group presents the highest levels of anxiety symptoms $(M=18.25, S D=12.49)$. A series of post-hoc tests using Bonferroni showed that the moderate exercise group $(M=23.47, S D=15.07)$ had significantly more anxiety than the high exercise group $(M=9.78, S D=7.71), p<0.05$. It also revealed that diabetes mellitus' patients $(M=18.25, S D=12.49)$ present significantly higher levels of anxiety than psoriasis' patients $(M=11.70, S D=9.69), p$ $<0.05$.

For depression, there is no main effect of exercise or medical condition. No interaction between the medical condition and physical activity was found. Lastly, for stress, there is no main effect of exercise or medical condition. No interaction between the medical condition and physical activity was found.

Figure 1 shows the distribution of anxiety per each type of disease. Diabetes mellitus group presents the highest levels of anxiety symptoms $(M=18.25, S D=$ 12.49).

Figure 2 shows the levels of anxiety per each exercise group. Participants that exercise actively ( 3 or 4 times per week) demonstrate the least symptoms of anxiety.

\subsection{Life Satisfaction}

There is no main effect of exercise or medical condition in life satisfaction. No

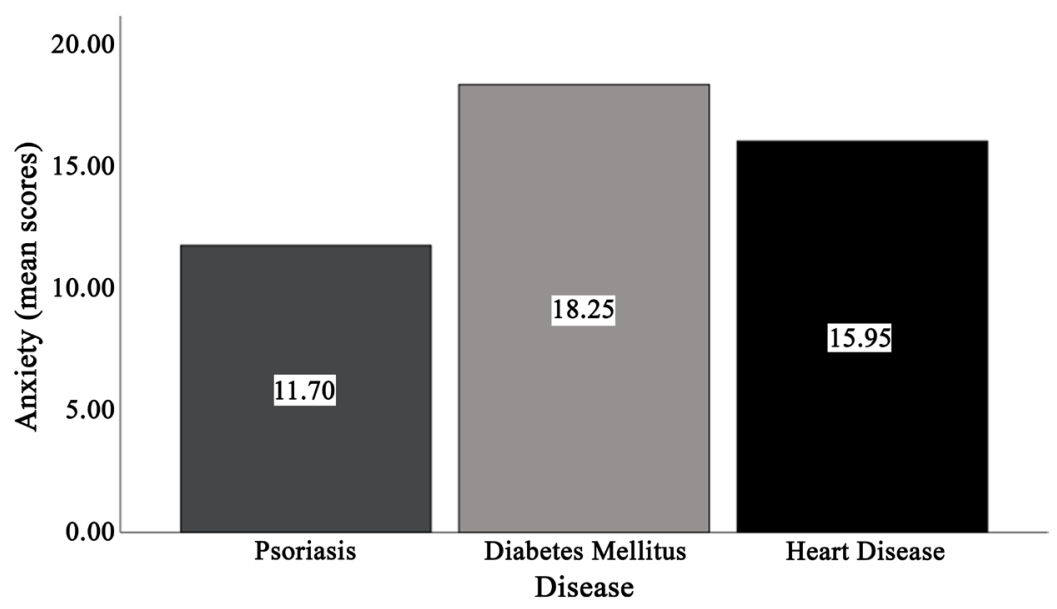

Figure 1. Anxiety per type of disease. 
interaction between the medical condition and physical activity was found.

\subsection{Subjective Happiness}

There is no main effect of exercise or medical condition in subjective happiness. No interaction between the medical condition and physical activity was found.

\subsection{Meaning in Life}

\section{Presence of Meaning in Life}

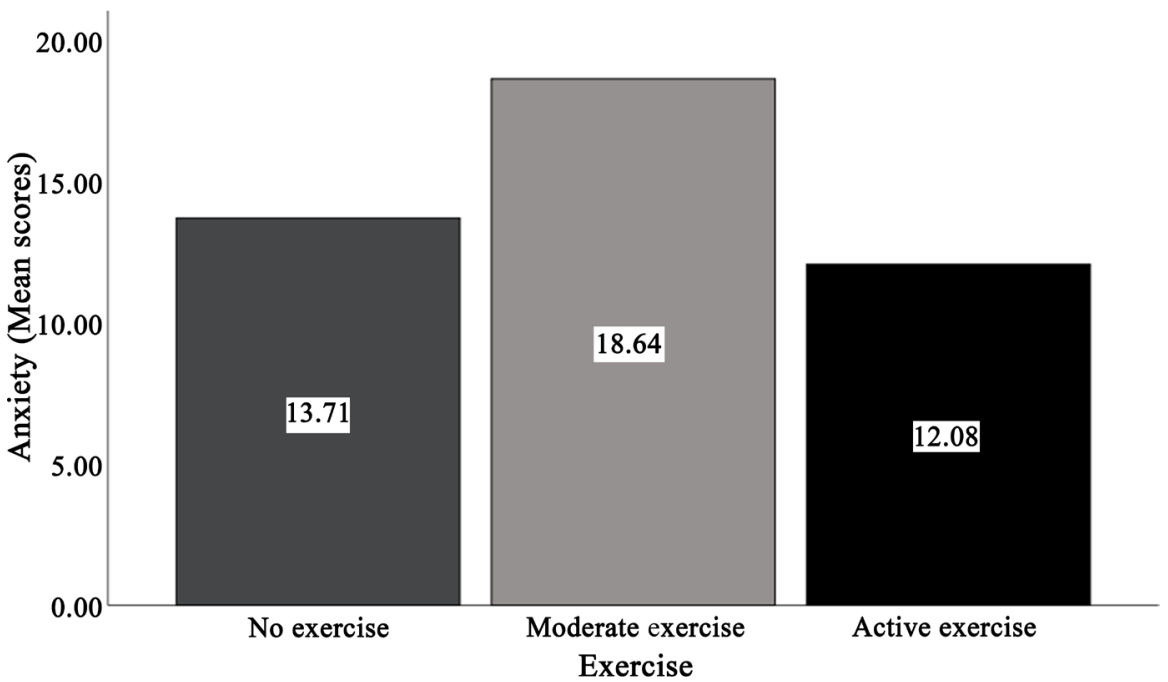

Figure 2. Anxiety per frequency of exercise.

Table 3. Means, standard deviations as a function of 3 (Exercise) $\times 3$ (Diseases) in Life Satisfaction.

\begin{tabular}{ccccccccccc}
\hline \multirow{2}{*}{ Exercise } & \multicolumn{3}{c}{ Psoriasis } & \multicolumn{3}{c}{ Diabetes } & \multicolumn{3}{c}{ Heart Disease } & \multicolumn{4}{c}{ ANOVA } \\
\cline { 2 - 11 } & $\mathbf{M}$ & SD & M & SD & M & SD & Effect & F & df & $\eta^{2}$ \\
\hline No & 21.94 & 8.97 & 16.75 & 8.11 & 21.47 & 8.97 & D & 0.420 & 2 & 0.008 \\
Moderate & 19.84 & 8.47 & 20.40 & 7.58 & 20.00 & 9.10 & E & 0.566 & 2 & 0.010 \\
High & 23.25 & 8.54 & 22.22 & 5.78 & 21.08 & 5.66 & D $\times$ E & 0.768 & 4 & 0.027 \\
\hline
\end{tabular}

Psoriasis participants, who exercise at a high frequency present the highest score in satisfaction of life $($ mean $=23.25, \mathrm{SD}=8.54)$.

Table 4. Means, standard deviations as a function of 3 (Exercise) $\times 3$ (Diseases) in Subjective Happiness.

\begin{tabular}{ccccccccccc}
\hline \multirow{2}{*}{ Exercise } & \multicolumn{3}{c}{ Psoriasis } & \multicolumn{2}{c}{ Diabetes } & \multicolumn{3}{c}{ Heart Disease } & \multicolumn{4}{c}{ ANOVA } \\
\cline { 2 - 11 } & $\mathbf{M}$ & SD & M & SD & M & SD & Effect & F & df & $\eta^{2}$ \\
\hline No & 16.59 & 4.33 & 15.37 & 5.61 & 17.53 & 4.47 & D & 0.330 & 2 & 0.006 \\
Moderate & 15.95 & 3.67 & 16.07 & 3.13 & 16.08 & 4.23 & E & 1.677 & 2 & 0.029 \\
High & 17.75 & 4.72 & 18.11 & 3.02 & 18.17 & 2.17 & $\mathrm{D} \times \mathrm{E}$ & 0.336 & 4 & 0.012 \\
\hline
\end{tabular}

Heart disease individuals (mean $=18.08, \mathrm{SD}=2.83$ ) at a high exercise frequency express the highest scores in subjective happiness. 


\section{Search of Meaning in Life}

Two $3 \times 3$ ANOVAs were conducted, after testing for their statistical assumptions. An interaction between medical condition and frequency of exercise was found $(p<0.05)$ in the presence of meaning in life value. The ANOVA showed that there is an interaction between exercise frequency and type of disease: $F(2$, $111)=3.241, p<0.05, \eta^{2}=0.105$. To calculate the post-hoc comparisons between the levels of the independent variables, a new variable was calculated: interaction of exercise frequency and medical condition. ANOVA results by one factor showed that diabetes mellitus group presented the greatest variance according to the frequency of exercise. Diabetes mellitus participants that do not exercise $(M=18.69, S D=6.96)$ present the lowest scores in the presence of meaning in life value, whereas those who adopt a moderate frequency $(M=$ $25.13, S D=6.32$ ) achieved the highest scores. On the other hand, psoriasis and heart disease individuals seem to achieve a decent score, even though they do not exercise. Those individuals, among diabetes mellitus patients, that exercise regularly expressed similar scores with the moderate frequency exercisers. There is no main effect of exercise or medical condition in search of meaning in life. No interaction between the exercise and the type of exercise was found. Figure 3 shows the interaction between physical activity, medical condition and presence of meaning in life.

\section{Discussion}

The main focus of the study was to investigate the potential effect of physical exercise and type of disease on NES and well-being measurements. Overall, results demonstrated differences regarding diabetes mellitus in anxiety, moderate exercise in anxiety and interaction between exercise and disease in the presence of meaning in life value. More concisely, participants that exercise at a moderate

Table 5. Means, standard deviations as a function of 3 (Exercise) $\times 3$ (Diseases) in the presence of Meaning in Life.

\begin{tabular}{cccccccccccc}
\hline \multirow{2}{*}{$\begin{array}{c}\text { Presence of MOL } \\
\text { Exercise }\end{array}$} & \multicolumn{2}{c}{ Psoriasis } & \multicolumn{2}{c}{ Diabetes } & \multicolumn{3}{c}{ Heart Disease } & \multicolumn{5}{c}{ ANOVA } \\
\cline { 2 - 11 } & $\mathbf{M}$ & SD & M & SD & M & SD & Effect & F & df & $\eta^{2}$ \\
\hline No & 22.47 & 7.74 & 18.69 & 6.96 & 24.40 & 4.92 & D & 0.118 & 2 & 0.002 \\
Moderate & 22.16 & 4.99 & 25.13 & 6.32 & 19.92 & 6.76 & E & 0.912 & 2 & 0.016 \\
High & 24.75 & 4.19 & 24.44 & 4.03 & 22.83 & 4.99 & D $\times$ E & $3.241^{\star}$ & 4 & 0.105 \\
\hline Search for MOL & & & & & & & & & & \\
Exercise & $\mathbf{M}$ & SD & M & SD & M & SD & Effect & F & df & $\eta^{2}$ \\
\hline No & 21.76 & 7.69 & 18.94 & 9.32 & 23.27 & 6.11 & D & 1.053 & 2 & 0.019 \\
Moderate & 22.89 & 6.02 & 22.33 & 4.97 & 19.69 & 9.21 & E & 1.184 & 2 & 0.021 \\
High & 26.00 & 3.91 & 26.00 & 4.47 & 20.17 & 5.24 & D $\times$ E & 1.967 & 4 & 0.066 \\
\hline
\end{tabular}

*Significant at the $p<0.05$ level. Psoriasis individuals (mean $=24.74, \mathrm{SD}=4.19$ ) at a high frequency of physical activity express higher scores in the presence of meaning in life value. 


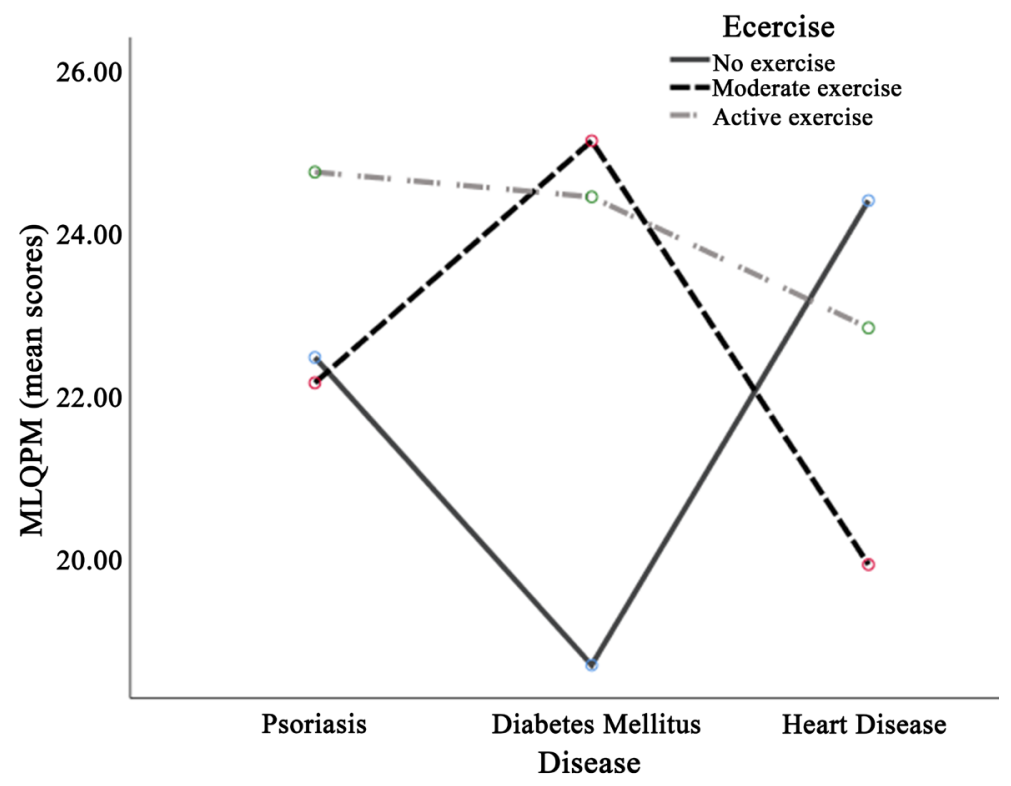

Figure 3. Interaction of exercise frequency, type of disease and presence of meaning in life (MLQPM).

frequency achieved the highest score in anxiety: psoriasis group, diabetes mellitus group, heart disease patients. The diabetes mellitus group also presents the highest score in anxiety among medical condition groups. Additionally, diabetes mellitus participants, who exercise at a moderate frequency presented the highest score in the purpose of meaning in life value. ANOVA did not show a significant result in depression, anxiety, life satisfaction, subjective happiness and search of meaning in life. The ANOVA showed that people who exercise at a moderate frequency present higher scores in anxiety. Secondly, diabetes mellitus patients express more anxiety among other medical condition groups. Furthermore, the same group scored higher in the purpose of meaning in life scale.

According to the first research hypothesis that NES will with the disease was confirmed: anxiety was revealed to increase concerning diabetes mellitus. The other variables did not present a significant difference. Additionally, no significant difference in well-being values in terms of the type of disease was found. The results of this study are concordant with previous findings. The casualty of exercise with anxiety confirms preceding evidence. Dunn, Trivedi, and O'Neal (2001) conducted a systematic study to present information about the intensity, duration and frequency of exercise prescription, in depressive and anxious symptoms. It was revealed that exercise does not present a significant reduction in anxiety. I is conducted a review study and demonstrated that the frequency of exercise does play an important role in anxiety reduction. Four or more times per week should be encouraged in order for anxiety levels to decrease. The main findings from the present research are that people who are physically active at a high frequency ( 1 or 2 times per week) present in generally higher levels of anxiety. This may be the result of not engaging in physical activity or engaging at a deficient level of exercise, which does not contribute to maintaining a decent 
fitness level. In the sample, the participants who exercised more expressed fewer anxious and depressive symptoms than the participants who exercised less. DeBoer, Powers, Utschig, Otto, and Smits (2012) conducted a review to explore the case that exercise might be useful for anxiety disorders. Results revealed that vigorous physical activity that is 3 or 4 times frequency per week for at least 15 minutes could decrease anxiety levels. In this study, there was a significant difference in NES in terms of vigorous exercise.

Analyses indicated that increases in exercise frequency did not predict decreases in anxious and depressive symptoms. Moderate physical activity (1 or 2 times per week) is related to increased anxious symptoms, but the association is not because of an interaction with a medical condition. This may be interpreted due to motivational or locus of control factors, that attribute to the reasons for which individuals may exercise. A study (Weinstein, Maayan and Weinstein, 2015) showed that compulsory exercise increases levels of anxiety, instead of demonstrating beneficial results. It might also be a result of the period that the participants reviewed the questionnaires. DASS 21, which measures the anxiety levels, refers to the expression of the symptoms in the previous week. Therefore, there are contradicting findings for the effect of exercise in anxiety.

In this study, no significant difference was revealed for depression. It was implied that no physical activity is correlated with depression. Moreover, it is reported that regular physical activity was related to higher social functioning. However, it is not correlated with lower levels of NES. This study presented no evidence that NES symptoms have a relationship with exercise. On the other hand, Dunn et al. (2001) presented evidence that depression decreases with physical activity.

In this study, three medical condition groups were investigated: diabetes mellitus, heart disease and psoriasis. From the latter, diabetes mellitus group expressed more anxiety among all the groups. It is known that diabetes mellitus patients have to engage in a sportive lifestyle in order to cope with their disease. Diabetes and its consequences can be treated, avoided or delayed with diet, physical activity, medication and regular screening and treatment for complications. As a result, exercise is a critical predictive factor in the progression of their health and diabetes mellitus patients have to deal with much pressure due to the nature of their medical condition. Thus, physical activity may contribute more to their anxiety levels, being compulsory (Weinstein, Maayan and Weinstein, 2015). Additionally, glycemic control may contribute more to anxiety in diabetes type 1 group of patients (Strandberg, Graue, Wentzel-Larsen, Peyrot and Rokne, 2014). Regarding diabetes, relationship status might also be important in their anxiety levels. It is supported that patients who live in an encouraging and supportive environment tend to focus more on following a healthy routine. Furthermore, their anxiety levels are lower, as they engage in physical activity willingly-at least 30 minutes of regular, moderate-intensity activity on most days. More activity is required for weight control. Tang and his colleagues (2008) also 
supported that social support plays a role in the diabetes-specific quality of life and self-management practices. Social support encompasses multiple dimensions that differentially influence specific diabetes health-related outcomes and behaviours.

The second hypothesis that there will be an interaction between exercise and type of disease in terms of NES or well-being was not fully confirmed. An interaction between exercise and type of disease in terms of the purpose of meaning in life value was reported. Alternative ways of self-regulation or the presence of acceptance in patients' lives might explainn the interaction. A study showed that well-being scores predict lower rates of exercise-stress induced myocardial ischemia in a cohort of patients with ischemic heart disease (Feigal, Boyle, Samad, Velazquez, Wilson, Becker, Williams, Kuhn, Ortel, Rogers, O'Connor, and Jiang, 2017). Pennedo and Dahn in 2005 reviewed research about the relationship between exercise, physical activity and physical and mental health. Results showed better health outcomes and better well-being values. Furthermore, Giannini, Mohn, \& Chiarelli (2006) reported that exercise might attribute to prevent chronic medical conditions and to improve psychological well-being. Myers, McVay, Brashear, Johannsen, Swift, Kramer, Harris, Johnson, Earnest and Church (2013) conducted a randomised control trial to demonstrate that physical activity has beneficial effects on well-being in patients with type 2 diabetes.

All in all, this study shows that there is a main effect of exercise in anxiety, a main effect of disease in anxiety and interaction of exercise and disease in the presence of meaning in life. No other main effect or interaction regarding the other dependent variables in negative emotional state or well-being values are revealed. Previous studies present contradicting findings regarding the relationship between exercise and anxiety. The relationship between diabetes and anxiety is confirmed. Finally, the interaction between physical activity and medical condition on the presence of meaning in life value is also confirmed.

\section{Implications}

The aim of the current study was to examine the effect physical activity and type of disease may have on negative emotional state and well-being among diabetes, heart disease and psoriasis population. The purpose was to increase the understanding regarding the role of exercise in chronic illnesses regarding the latter variables in adulthood. A better understanding of the role of exercise could help researchers and therapists to design appropriate intervention plans to treat anxiety and enhance well-being in specific diseases. Physical activity is a way everyone can incorporate in their lives, which is essential for intervention planning. The findings demonstrated that diabetes patients exhibit higher levels of anxiety compared to psoriasis or heart disease population. This indicates that the specific group may probably benefit the most of such an intervention. However, the other two groups should not be marginalised. Psoriasis patients appear to avoid 
exercise due to disease-related issues, such as their skin appearance, whereas heart disease patients appear to avoid physical activity due to health impairments. Therefore, it is essential to promote exercise among these groups to investigate whether they may also benefit.

This research may also contribute to improving well-being in people's life. It is also important to note that it may add to the practical application of positive health and decisive intervention. Positive health refers to taking care of physical and mental health, which relates to higher energy levels and self-confidence, thus a more active lifestyle. Furthermore, it may provide individuals with the ability to enhance more positively their life. The implement of a focused and well-established intervention program may provide individuals with the chance to improve their psychological and mental state.

Moreover, through the recording of their needs, it may be easier to propose more effective ways to fulfil them. Therefore, mental health prevention may also be enhanced. The current research consists of a stimulus to study diverse disease groups, so that implementation of specific interventions may be applied. To conclude, according to the findings there is a need to develop intervention plans targeting diabetes, psoriasis and heart disease groups, aiming at increasing well-being and reduce negative emotional state by incorporating physical activity in the intervention planning.

\section{Limitations and Future Research Directions}

This research presented some limitations. Sample size limited the implications of the benefits associated with physical activity. Disease-related problems which limited the available sample and the low motive to exercise were a few of them. Self-report questionnaires could also be another factor of limitation. Self-report measurements are often biased as participants tend to provide socially accepted responses (Howell, 2013). At this initial stage, it is suggested that a later longitude study may prove beneficial for the outcomes of the research. Future studies may be guided towards a larger sample with the collaboration of relevant organisations and hospitals, so that possible positive results may be investigated more thoroughly. Consistency of exercise and other aspects such as intensity and duration could also be further investigated in repeated-measures research designs. Furthermore, more types of susceptible population could be investigated as well.

This study and previous literature revealed that there is a relationship between the frequency of exercise and physical activity with a negative emotional state. The same applies to well-being values. Additionally, the type of exercise would be another interesting factor to investigate. Myers et al. (2013) supported that mixed exercise type presents the most beneficial effects on diabetes patients. Therefore, which type of training contributes more to each variable could be another aspect of interest.

In conclusion, physical activity is considered to have a positive outcome to 


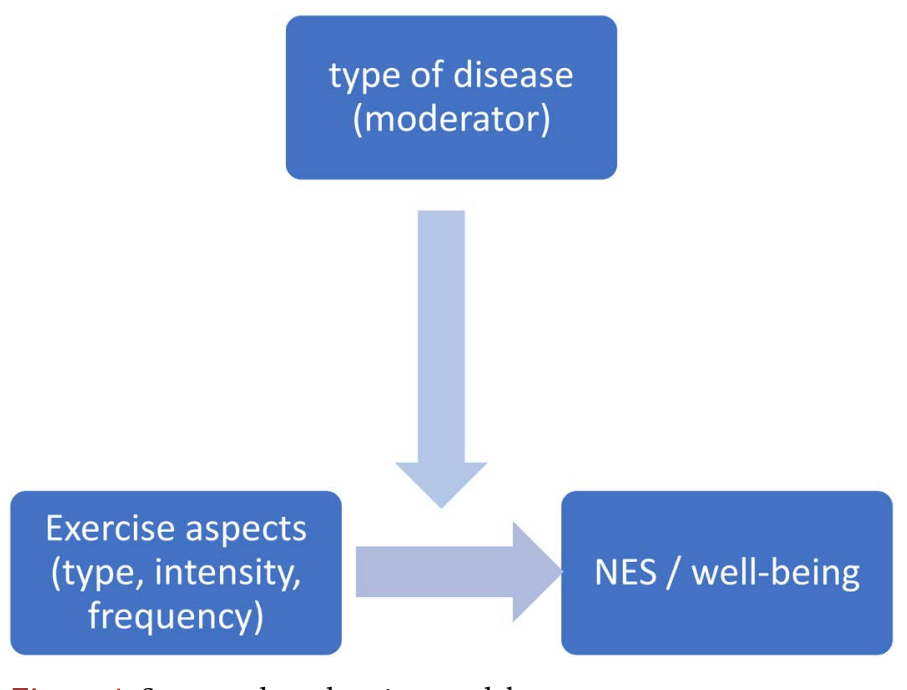

Figure 4. Suggested moderation model.

general well-being and regulation of negative emotional state (Penedo and Dahn, 2005). The results indicated that the moderate frequency of physical activity could increase anxiety. The same applies to a medical condition and especially, to diabetes mellitus. Furthermore, there is an interaction between exercise and disease in terms of well-being values, that is the presence of meaning in life. The results are following previous findings: physical activity promotes physical and mental well-being. Additionally, the result extended the literature because they showed the interaction between disease and training. As a result, it is suggested that future studies should address this issue in more details.

In the current study, the type of disease and exercise frequency were manipulated as independent variables. The researchers investigated their possible effect on NES and well-being. A moderation model is suggested for future research. Future research could be guided toward handling type of disease as a moderator (Figure 4). Future researchers could study further exercise aspects, such as type of exercise, the intensity of exercise and frequency of exercise.

In conclusion, physical activity is considered to have a positive outcome to general well-being and regulation of negative emotional state (Penedo and Dahn, 2005). The results indicated that the moderate frequency of physical activity could increase anxiety. The same applies to a medical condition and especially, to diabetes mellitus. Furthermore, there is an interaction between exercise and disease in terms of well-being values that is the presence of meaning in life. The results are following previous findings: physical activity promotes physical and mental well-being. Additionally, the result extended the literature because they showed the interaction between disease and training. As a result, it is suggested that future studies should address this issue in more detail.

\section{Conflicts of Interest}

The authors declare no conflicts of interest regarding the publication of this paper. 


\section{References}

Bernstein, G. A., \& Borchardt, C. M. (1991). Anxiety Disorders of Childhood and Adolescence: A Critical Review. Journal of the American Academy of Child \& Adolescent Psychiatry, 30, 519-532. https://doi.org/10.1097/00004583-199107000-00001

Cohen, R. A. (2011). Yerkes-Dodson Law. In J. S. Kreutzer, J. DeLuca, \& B. Caplan (Eds.), Encyclopedia of Clinical Neuropsychology. New York: Springer. https://doi.org/10.1007/978-0-387-79948-3_1340

Cohen, S., \& Rodriguez, R. (1995). Pathways Linking Affective Disturbances and Physical Disorders. Health Psychology, 15, 374-380. https://doi.org/10.1037/0278-6133.14.5.374

Cotman, C. W., \& Berchtold, N. C. (2002). Exercise: A Behavioral Intervention to Enhance Brain Health and Plasticity. Trends in Neurosciences, 25, 295-301. https://doi.org/10.1016/S0166-2236(02)02143-4

Cunliffe, W. J., Holubar, K., Millikan, L. E., Parish, L. C., Ring, J., Lipozenèiæ, J. et al. (2003). Topical Procedures, Innovations, and Mistreatments in Dermatovenerology. Acta Dermatovenerologica Croatica, 11, 89.

De Arruda, L. H. F., \& De Moraes, A. P. F. (2001). The Impact of Psoriasis on Quality of Life. British Journal of Dermatology, 144, 33-36. https://doi.org/10.1046/j.1365-2133.2001.144s58033.x

De Moor, M. H. M., Beem, A. L., Stubbe, J. H., Boomsma, D. I., \& De Geus, E. J. C. (2006). Regular Exercise, Anxiety, Depression and Personality: A Population-Based Study. Preventive Medicine, 42, 273-279. https://doi.org/10.1016/j.ypmed.2005.12.002

De Moor, M. H. M., Boomsma, D. I., Stubbe, J. H., Willemsen, G., \& de Geus, E. J. C. (2008). Testing Causality in the Association between Regular Exercise and Symptoms of Anxiety and Depression. Archives of General Psychiatry, 65, 897-905. https://doi.org/10.1001/archpsyc.65.8.897

DeBoer, L. B., Powers, M. B., Utschig, A. C., Otto, M. W., \& Smits, J. A. J. (2012). Exploring Exercise as an Avenue for the Treatment of Anxiety Disorders. Expert Review of Neurotherapeutics, 12, 1011-1022. https://doi.org/10.1586/ern.12.73

Dunn, A. L., Trivedi, M. H., \& O’Neal, H. A. (2001). Physical Activity Dose-Response Effects on Outcomes of Depression and Anxiety. Medicine and Science in Sports and EXercise, 33, 587-S597. https://doi.org/10.1097/00005768-200106001-00027

Epps, F., To, H., Liu, T. T., Karanjit, A., \& Warren, G. (2019). Effect of Exercise Training on the Mental and Physical Well-Being of Caregivers for Persons Living with Chronic Illnesses: A Systematic Review and Meta-Analysis. Journal of Applied Gerontology, 0733464819890753. https://doi.org/10.1177/0733464819890753

Evans, M., Rohan, K. J., Howard, A., Ho, S. Y., Dubbert, P. M., \& Stetson, B. A. (2017). Exercise Dimensions and Psychological Well-Being: A Community-Based Exercise Study. Journal of Clinical Sport Psychology, 11, 107-125.

https://doi.org/10.1123/jcsp.2017-0027

Feigal, J. P., Boyle, S. H., Samad, Z., Velazquez, E. J., Wilson, J. L., Becker, R. C., Williams Jr., R. B., Kuhn, C. M., Ortel, T. L., Rogers, J. G., O’Connor, C. M., \& Jiang, W. (2017). Associations between Positive Emotional Well-Being and Stress-Induced Myocardial Ischemia: Well-Being Scores Predict Exercise-Induced Ischemia. Psychosomatic Research, 93, 14-18. https://doi.org/10.1016/j.jpsychores.2016.11.012

Galanakis, M., Lakioti, A., Pezirkianidis, C., Karakasidou, E., \& Stalikas, A. (2017). Reliability and Validity of the Satisfaction with Life Scale (SWLS) in a Greek Sample. International Journal of Humanities and Social Studies, 5, 120-127.

Genuth, S., Alberti, K. G., Bennett P., Buse, J., Defronzo, R., Kahn, R., Kitzmiller, J., 
Knowler, W. C., Lebovitz, H., Lernmark, A., Nathan, D., Palmer, J., Rizza, R., Saudek, C., Shaw, J., Steffes, M., Stern, M., Tuomilehto, J., \& Zimmet, P. (2003). Expert Committee on the Diagnosis and Classification of Diabetes Mellitus 2, the Expert Committee on the Diagnosis and Classification of Diabetes Mellitus. Follow-Up Report on the Diagnosis of Diabetes Mellitus. Diabetes Care, 26, 3160-3167.

https://doi.org/10.2337/diacare.26.11.3160

Giannini, C., Mohn, A., \& Chiarelli, F. (2006). Physical Exercise and Diabetes during Childhood. Acta Bio-Medica, 77, 18-25.

Gordon, B. R., McDowell, C. P., Lyons, M., \& Herring, M. P. (2017). The Effects of Resistance Exercise Training on Anxiety: A Meta-Analysis and Meta-Regression Analysis of Randomised Controlled Trials. Sports Medicine, 47, 2521-2532. https://doi.org/10.1007/s40279-017-0769-0

Hassmén, P., Koivula, N., \& Uutela, A. (2000). Physical Exercise and Psychological Well-Being: A Population Study in Finland. Preventive Medicine, 30, 17-25. https://doi.org/10.1006/pmed.1999.0597

Herring, M. P., O’Connor, P. J., \& Dishman, R. K. (2010). The Effect of Exercise Training on Anxiety Symptoms among Patients: A Systematic Review. Archives of Internal Medicine, 170, 321-331. https://doi.org/10.1001/archinternmed.2009.530

Howell, D. (2013). Fundamental Statistics for the Behavioral Sciences. Boston, MA: Cengage Learning.

Huebschmann, A. G., Schauer, I. E., Bauer, T. A., Regensteiner, J. G., \& Reusch, J. E. (2018). Exercise Performance Impairments and Benefits of Exercise Training in Diabetes. In Diabetes and Exercise (pp. 83-108). Cham: Humana Press.

https://doi.org/10.1007/978-3-319-61013-9_6

International Diabetes Foundation (2020).

https://idf.org/our-network/regions-members/europe/members/137-greece.html

Ivanova, E., Burns, R. J., Deschênes, S. S., Knäuper, B., \& Schmitz, N. (2017). A Longitudinal Investigation of Anxiety and Depressive Symptomatology and Exercise Behaviour among Adults with Type 2 Diabetes Mellitus. Canadian Journal of Diabetes, 41, 73-81. https://doi.org/10.1016/j.jcjd.2016.07.006

Jayakody, K., Gunadasa, S., \& Hosker, C. (2014). Exercise for Anxiety Disorders: Systematic Review. British Journal of Sports Medicine, 48, 187-196.

https://doi.org/10.1136/bjsports-2012-091287

Karakasidou, E., Pezirkianidis, C., Stalikas, A., \& Galanakis, M. (2016). Standardisation of the Subjective Happiness Scale (SHS) in a Greek Sample. Psychology, 7, 1753-1765. https://doi.org/10.4236/psych.2016.714164

Liu, T., Chan, A. W., Liu, Y. H., \& Taylor-Piliae, R. E. (2018). Effects of Tai Chi-Based Cardiac Rehabilitation on Aerobic Endurance, Psychosocial Well-Being, and Cardiovascular Risk Reduction among Patients with Coronary Heart Disease: A Systematic Review and Meta-Analysis. European Journal of Cardiovascular Nursing, 17, 368-383. https://doi.org/10.1177/1474515117749592

Lovibond, P. F., \& Lovibond, S. H. (1995). The Structure of Negative Emotional States: Comparison of the Depression Anxiety Stress Scales (DASS) with the Beck Depression and Anxiety Inventories. Behaviour Research and Therapy, 33, 335-343. https://doi.org/10.1016/0005-7967(94)00075-U

Lyubomirsky, S., \& Lepper, H. (1999). A Measure of Subjective Happiness: Preliminary Reliability and Construct Validation. Social Indicators Research, 46, 137-155.

https://doi.org/10.1023/A:1006824100041

Mandolesi, L., Polverino, A., Montuori, S., Foti, F., Ferraioli, G., Sorrentino, P., \& Sorren- 
tino, G. (2018). Effects of Physical Exercise on Cognitive Functioning and Wellbeing: Biological and Psychological Benefits. Frontiers in Psychology, 9, 509.

https://doi.org/10.3389/fpsyg.2018.00509

Marquez, D. X., Jerome, G. J., McAuley, E., Snook, E. M., \& Canaklisova, S. (2010). Self-Efficacy Manipulation and State Anxiety Responses to Exercise in Low Active Women. Psychology \& Health, 17, 783-791. https://doi.org/10.1080/0887044021000054782

McDonough, E., Ayearst, R., Eder, L., Chandran, V., Rosen, C. F., Thavaneswaran, A., \& Gladman, D. D. (2014). Depression and Anxiety in Psoriatic Disease: Prevalence and Associated Factors. The Journal of Rheumatology, 41, 887-896. https://doi.org/10.3899/jrheum.130797

Michas, G., Karvelas, G., \& Trikas, A. (2019). Cardiovascular Disease in Greece; the Latest Evidence on Risk Factors. Hellenic Journal of Cardiology, 60, 271-275.

https://doi.org/10.1016/j.hjc.2018.09.006

Myers, V. H., McVay, M. A., Brashear, M. M., Johannsen, N. M., Swift, D. L., Kramer, K., Harris, M. N., Johnson, W. D., Earnest, C. P., \& Church, T. S. (2013). Exercise Training and Quality of Life in Individuals with Type 2 Diabetes. American Diabetes Association, 36, 1884-1890. https://doi.org/10.2337/dc12-1153

National Psoriasis Foundation (2020). https://www.psoriasis.org/content/statistics

Penedo, F. J., \& Dahn, J. R. (2005). Exercise and Well-Being: A Review of Mental and Physical Health Benefits Associated with Physical Activity. Current Opinion in Psychiatry, 18, 189-193. https://doi.org/10.1097/00001504-200503000-00013

Pezirkianidis, C., Galanakis, M., Karakasidou, I., \& Stalikas, A. (2016). Validation of the Meaning in Life Questionnaire (MLQ) in a Greek Sample. Psychology, 7, 1518-1530. https://doi.org/10.4236/psych.2016.713148

Pezirkianidis, C., Karakasidou, E., Lakioti, A., Stalikas, A., \& Galanakis, M. (2018). Psychometric Properties of the Depression, Anxiety, StressScales-21 (DASS-21) in a Greek Sample. Psychology, 9, 2933-2950. https://doi.org/10.4236/psych.2018.915170

Reid, R. D., Tulloch, H. E., Sigal, R. J., Kenny, G. P., Fortier, M., McDonnell, L. et al. (2010). Effects of Aerobic Exercise, Resistance Exercise or Both, on Patient-Reported Health Status and Well-Being in Type 2 Diabetes Mellitus: A Randomised Trial. Diabetologia, 53, 632-640. https://doi.org/10.1007/s00125-009-1631-1

Rogerson, M. C., Murphy, B. M., Bird, S., \& Morris, T. (2012). "I Don’t Have the Heart": A Qualitative Study of Barriers to and Facilitators of Physical Activity for People with coronary Heart Disease and Depressive Symptoms. International Journal of Behavioral Nutrition and Physical Activity, 9, Article No. 140.

https://doi.org/10.1186/1479-5868-9-140

Rogerson, M., Wood, C., Pretty, J., Schoenmakers, P., Bloomfield, D., \& Barton, J. (2020). Regular Doses of Nature: The Efficacy of Green Exercise Interventions for Mental Wellbeing. International Journal of Environmental Research and Public Health, 17, 1526. https://doi.org/10.3390/ijerph17051526

Scully, D., Kremer, J., Meade, M. M., Graham, R., \& Dudgeon, K. (1998). Physical Exercise and Psychological Well-Being: A Critical Review. British Journal of Sports Medicine, 32, 111-120. https://doi.org/10.1136/bjsm.32.2.111

Steger, M. F., Frazier, P., Oishi, S., \& Kaler, M., (2006). The Meaning in Life Questionnaire: Assessing the Presence of and Search for Meaning in Life. Journal of Counseling Psychology, 53, 80-93. https://doi.org/10.1037/0022-0167.53.1.80

Strandberg, R. B., Graue, M., Wentzel-Larsen, T., Peyrot, M., \& Rokne, B. (2014). Relationships of Diabetes-Specific Emotional Distress, Depression, Anxiety, and Overall 
Well-Being with $\mathrm{HbA}_{1 \mathrm{c}}$ in Adult Persons with Type 1 Diabetes. Journal of Psychosomatic Research, 77, 174-179. https://doi.org/10.1016/j.jpsychores.2014.06.015

Ströhle, A. J. (2009). Physical Activity, Exercise, Depression and Anxiety Disorders. Journal of Neural Transmission, 116, Article No. 777. https://doi.org/10.1007/s00702-008-0092-x

Stubbs, B., Vancampfort, D., Rosenbaum, S., Firth, J., Cosco, T., Veronese, N., Salum, G. A., \& Schuch, F. B. (2017). An Examination of the Anxiolytic Effects of Exercise for People with Anxiety and Stress-Related Disorders: A Meta-Analysis. Psychiatry Research, 249, 102-108. https://doi.org/10.1016/j.psychres.2016.12.020

Tang, T. S., Brown, M. B., Funnell, M. M., \& Anderson, R. M. (2008). Social Support, Quality of Life, and Self-Care Behaviors among African Americans with Type 2 Diabetes. The Diabetes Educator, 34, 266-276. https://doi.org/10.1177/0145721708315680

Torres, T., Alexandre, J. M., Mendonça, D., Vasconcelos, C., Silva, B. M., \& Selores, M. (2014). Levels of Physical Activity in Patients with Severe Psoriasis: A Cross-Sectional Questionnaire Study. American Journal of Clinical Dermatology, 15, 129-135. https://doi.org/10.1007/s40257-014-0061-0

van der Mheen, M., van Beynum, I. M., Dulfer, K., van der Ende, J., van Galen, E., Duvekot, J. et al. (2018). The CHIP-Family Study to Improve the Psychosocial Wellbeing of Young Children with Congenital Heart Disease and Their Families: Design of a Randomised Controlled Trial. BMC Pediatrics, 18, Article No. 230. https://doi.org/10.1186/s12887-018-1183-y

Van Voorhees, A. S., \& Fried, R. (2009). Depression and Quality of Life in Psoriasis. Postgraduate Medicine, 121, 154-161. https://doi.org/10.3810/pgm.2009.07.2040

Weinstein, A., Maayan, G., \& Weinstein, Y. (2015). A Study on the Relationship between Compulsive Exercise, Depression and Anxiety. Journal of Behavioral Addictions, 4, 315-318. https://doi.org/10.1556/2006.4.2015.034

Whooley, M. A., De Jonge, P., Vittinghoff, E., Otte, C., Moos, R., Carney, R. M. et al. (2008). Depressive Symptoms, Health Behaviors, and Risk of Cardiovascular Events in Patients with Coronary Heart Disease. JAMA, 300, 2379-2388.

https://doi.org/10.1001/jama.2008.711 